Thorax (1967), 22. 437.

\title{
Acute Wegener's granulomatosis
}

\author{
DAVID LEAK AND G.P. CLEIN \\ From King's College Hospital, London, and the Department of Medicine, University of Cambridge
}

Two cases of acute Wegener's granulomatosis are described. The first ended fatally after an illness of only two weeks. In the second a remission of three years has been obtained with heavy doses of steroid. At present there is evidence of impaired pulmonary gas transfer and also chronic glomerulonephritis, but the patient is asymptomatic. When the disease presents with pulmonary cavitation it may be confused with pulmonary neoplasm, tuberculosis, or lung abscess, and the diagnosis is made by biopsy. The Rose-Waaler was strongly positive in the case tested.

In middle-aged patients presenting with haemoptysis, purulent sputum, fever, and the rapid development of cavitation in the lungs, the differ. ential diagnosis usually rests between bronchial neoplasm, pulmonary tuberculosis, lung abscess, and pulmonary infarction. Occasionally such an acute illness may be due to an acute pulmonary arteritis which would fulfil the criteria for Wegener's granulomatosis, a condition manifest by necrotizing granulomata in the respiratory tract, generalized vasculitis, and focal glomerulitis (Wegener, 1936 ; Godman and Churg, 1954). This illness generally lasts for a period of months or years (Walton, 1958) and terminates with chronic renal failure. However, it may occasionally run a rapid and fulminating course and present extreme difficulty in diagnosis. In view of the difficulty in diagnosis in such circumstances and the necessity of instituting treatment with steroids as early as possible, two cases are reported. The first ended fatally and undiagnosed after an illness of only two weeks. In the second, which at the onset was almost as acute, a diagnosis was made and a four years' remission has been obtained with large doses of prednisone.

\section{CASE HISTORIES}

CASE 1 Mrs. M.Y. aged 61 , previously quite fit apart from vague muscular pains for three to four weeks, suddenly developed rigors whilst attending a wedding. The next day she started to cough some heavily blood-stained sputum. Her temperature rose to $101^{\circ} \mathrm{F}$. She was treated at home for pneumonia with tetracycline. She failed to improve and after three days a chest radiograph showed consolidation of the right middle lobe and some patchy shadowing in the left lower zone. Treatment was changed to chloramphenicol and on the ninth day of the illness she was transferred to hospital.
On admission she was ill and afebrile, and was developing early peripheral circulatory failure. The pulse rate was $120 / \mathrm{min}$., the skin was cold and clammy, and the blood pressure $90 / 60 \mathrm{~mm}$. $\mathrm{Hg}$. There were signs of consolidation in the right middle lobe and her radiograph (Fig. 1) showed extension of the lesion in the right middle lobe with evidence of cavitation. Peripheral nodular shadows were present. The urine contained albumin and a few red cells but otherwise no deposit. Haemoglobin 12 g./100 ml., W.B.C. $17,000 /$ c.mm., $82 \%$ polymorphs. A blood film showed toxic granulation and a shift to the left of the polymorphs. There was no eosinophilia ; E.S.R. (Westergren) $32 \mathrm{~mm}$. in one hour; blood urea $42 \mathrm{mg} . / 100$ $\mathrm{ml}$. She was coughing large quantities of thick, heavily blood-stained sputum which was cultured on 10 occasions and was sterile. Gram and Ziehl-Neelsen smears were negative and cytological analysis was reported as showing a few cells suspicious of alveolarcell carcinoma. An E.C.G. showed low voltage complexes and flat T waves in V4 to 6 .

She was treated with penicillin, streptomycin, and prednisone, $15 \mathrm{mg}$. daily. On the fourth day following admission, two weeks from the onset of the illness, her blood pressure became unrecordable and despite standard resuscitative measures she died.

At necropsy there was some free fluid in the chest. In the lungs there were multiple peripheral nodules which were round or wedge-shaped, varying in size from $1 \mathrm{~cm}$. to $5 \mathrm{~cm}$. across. They were yellowish in colour and the largest in the right middle lobe was necrotic in the centre. There was a severe tracheobronchitis with much blood-stained mucopus. The spleen was twice normal size. The kidneys were (macroscopically) normal. The uterus was enlarged and the endometrium haemorrhagic. The upper respiratory tract was normal.

Histology showed an acute arteritis affecting the smaller arteries and arterioles. This was particularly florid in the lungs but was also present in the kidneys (Fig. 2), spleen, and uterus. In the lung, the nodules were found to consist of a central necrotic area surrounded by a chronic inflammatory cell infiltrate 

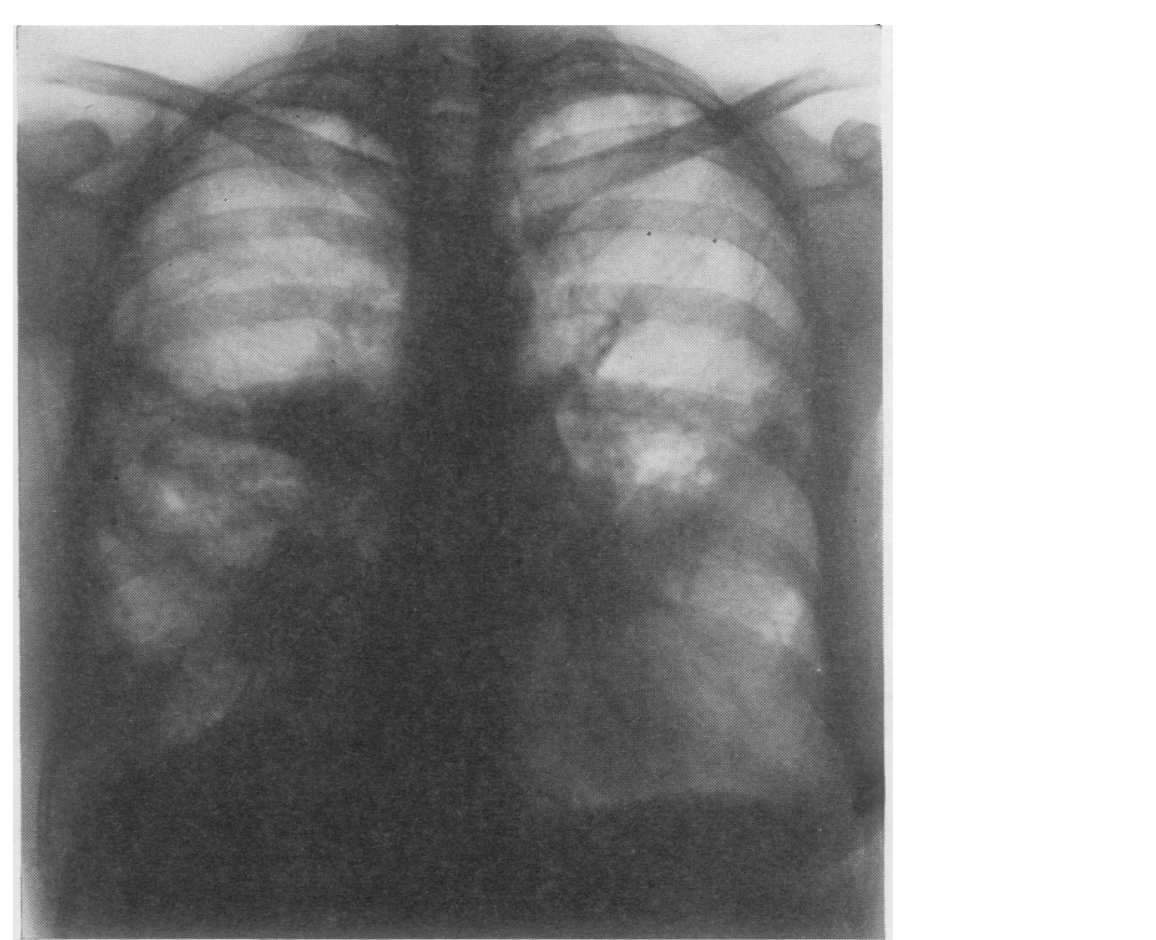

FIG. 1. Case 1. Chest radiograph. Extensive infiltration of right mid-zone
with evidence of cavitation. Peripheral nodular shadows in both lung fields.

FIG. 2. Case 1. Histology of the kidney. Panarteritis in a vessel of interlobular size. H. and E. $\times 80$.

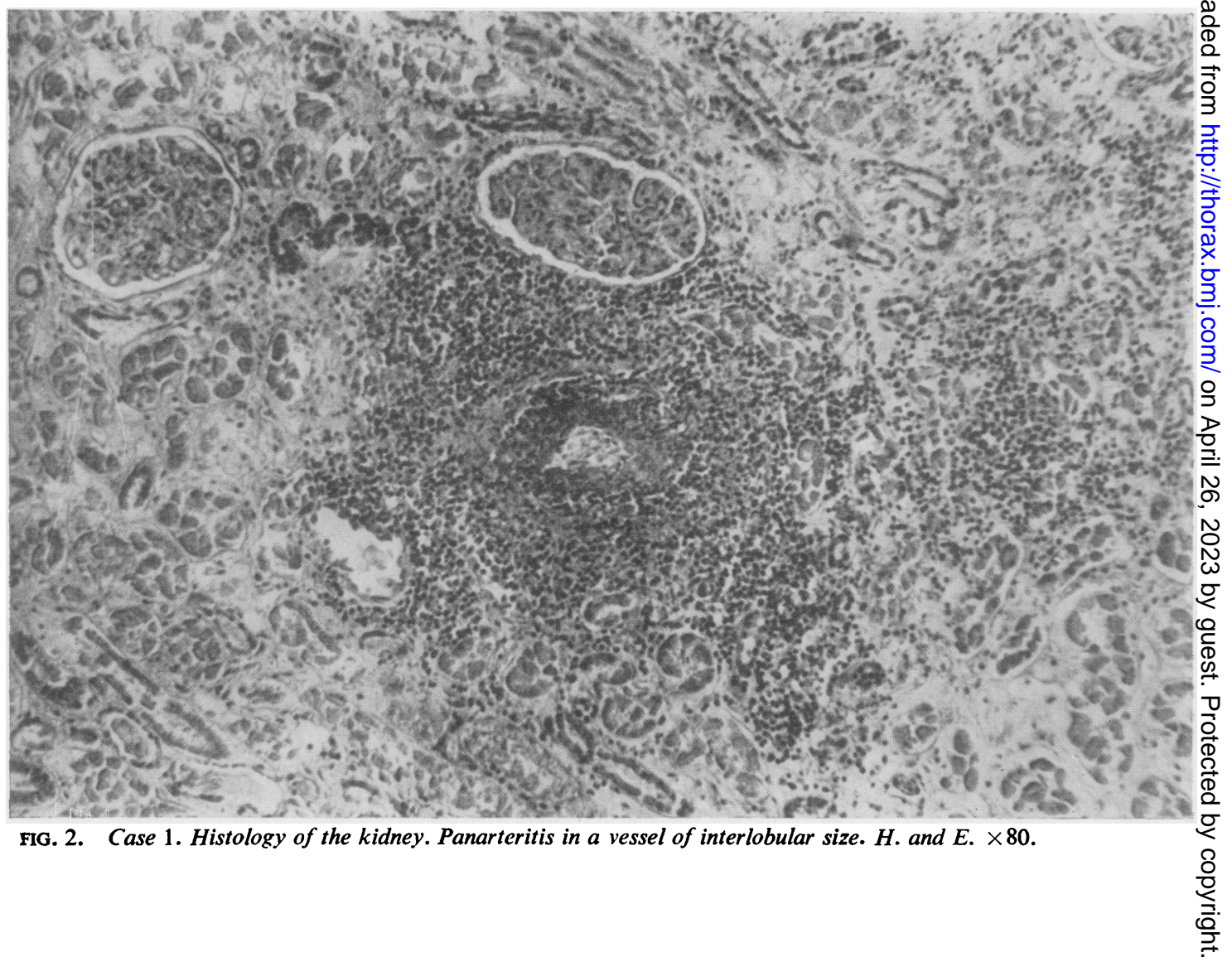




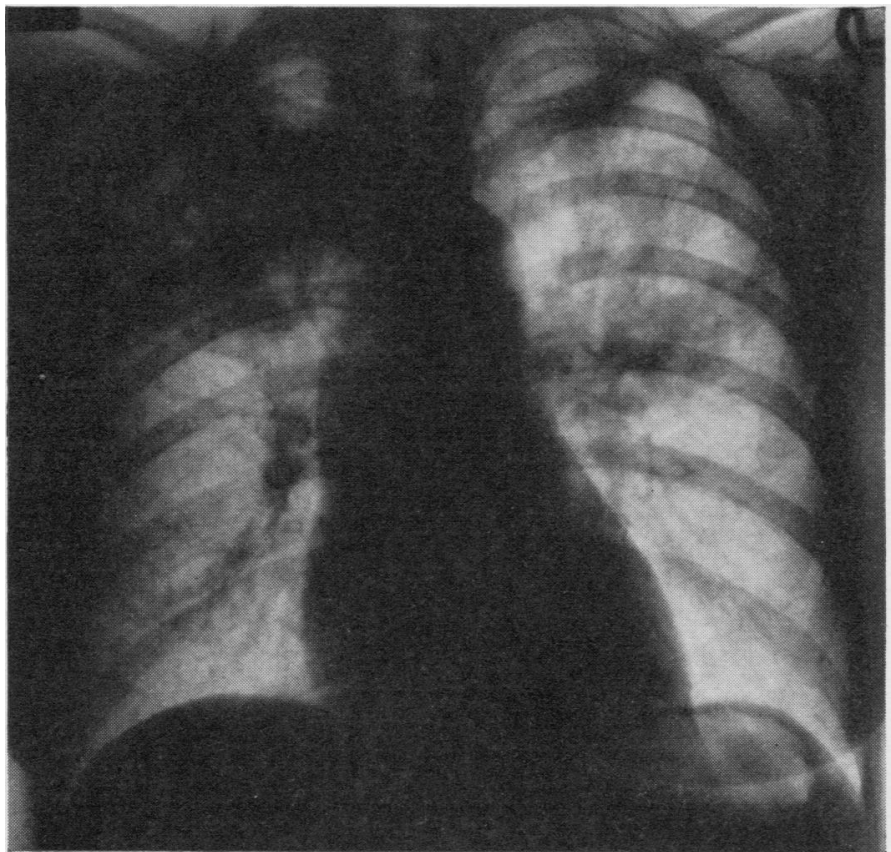

FIG. 3. Case 2. Chest radiograph. Large cavity with fluid level at right apex. Extensive infiltration of right upper lobe. Moderate infiltration in left upper lobe.

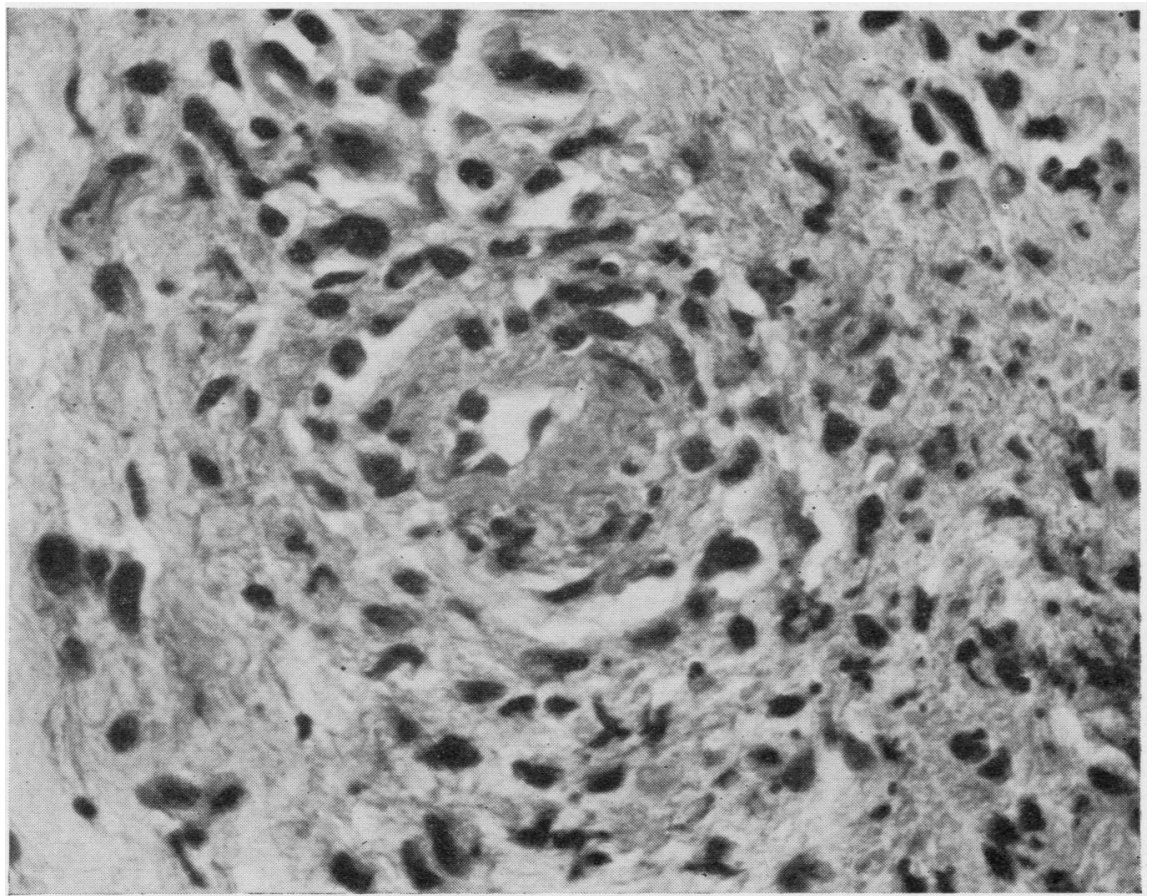

FIG. 4. Case 2. Skin biopsy. Necrotizing arteritis of small dermal artery. $H$. and $E . \times 550$. 


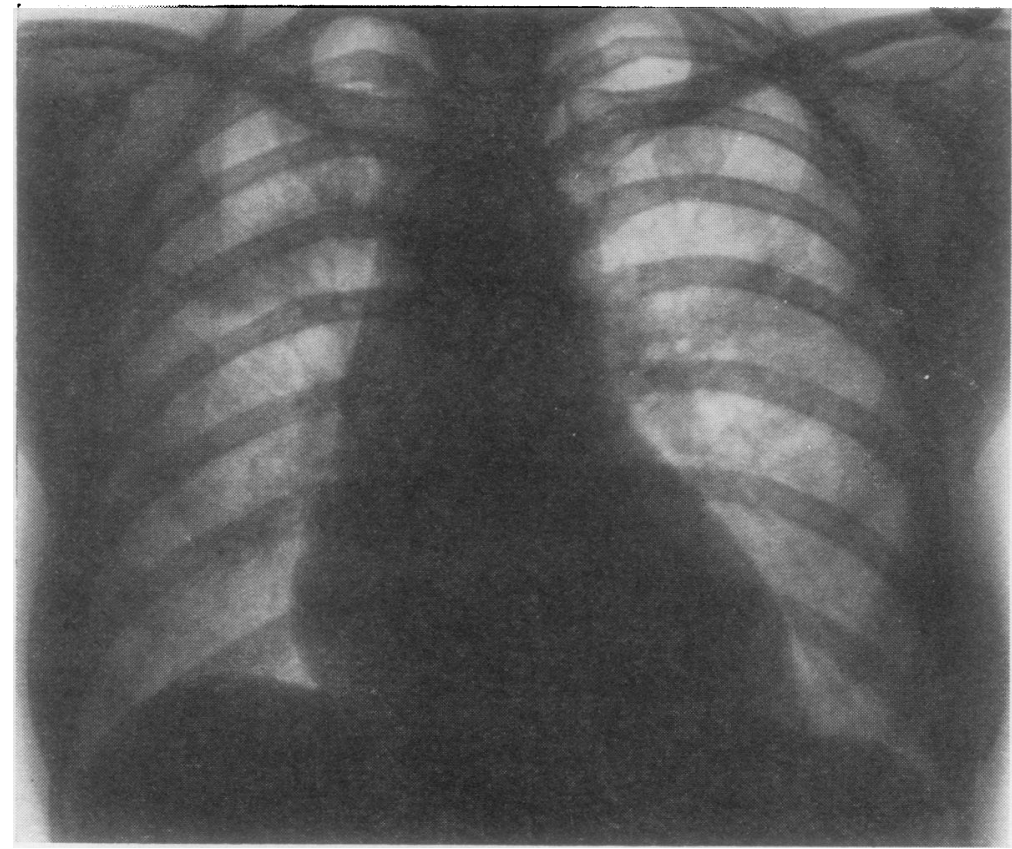

FIG. 5. Case 2. Chest radiograph after controi of acute illness with steroids. Infiltration of upper zones almost completely resolved. Cavity closed.

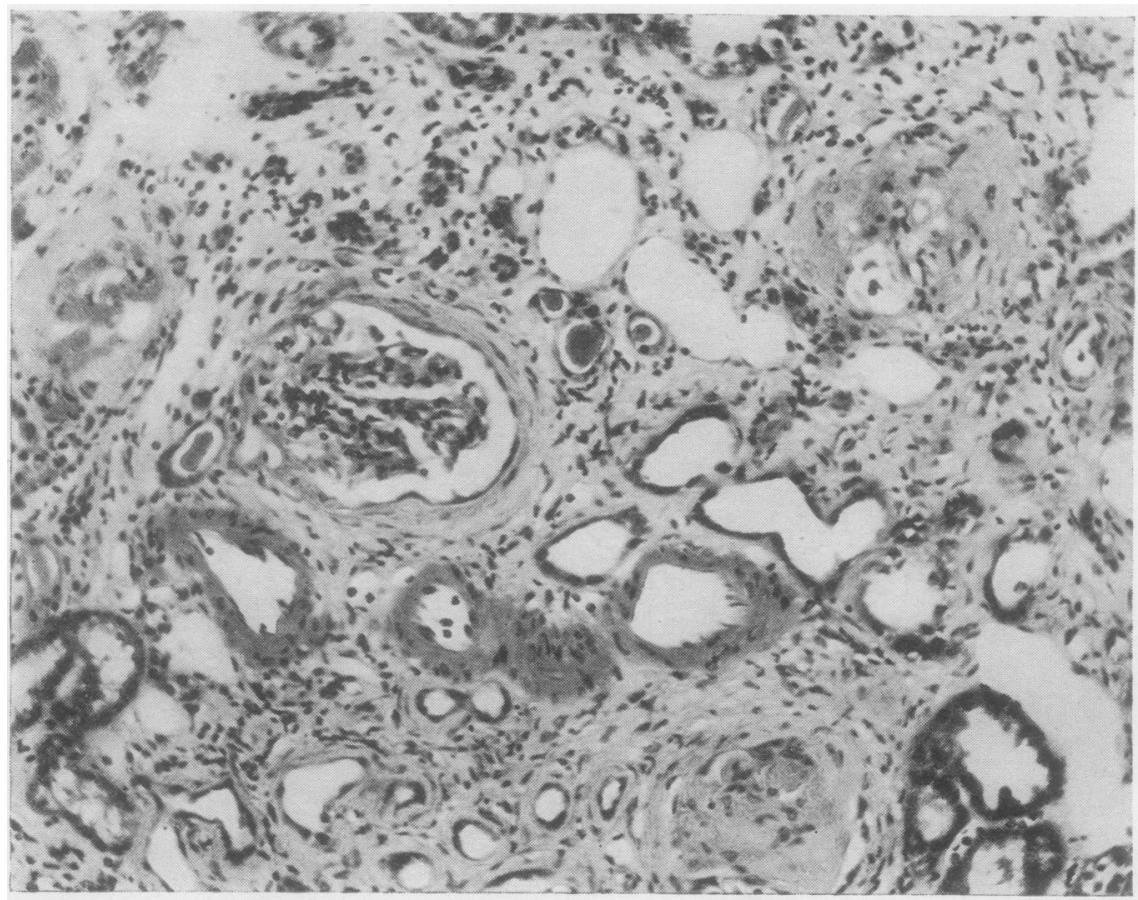

FIG. 6. Case 2. Renal biopsy taken after two years' steroid therapy. Chronic glomerulonephritis. H. and $E . \times 120$. 
which included giant cells. As in the other organs, the small arteries showed a panarteritis. In the kidney there were also a few areas of focal glomerulitis.

CASE 2 Mrs. E. H. aged 45, previously well, presented with vague stiffness of the knees and elbows of about five to six weeks' duration. Two small haemoptyses were followed by left-sided pleuritic pain four weeks before admission. Two days before admission she became suddenly ill with night sweats and severe haemoptysis.

Physical examination revealed an ill, toxic woman. Her temperature was $101^{\circ} \mathrm{F}$., pulse rate $108 / \mathrm{min}$., blood pressure $115 / 70 \mathrm{~mm}$. Hg. There were crepitations over the left upper lobe and stiffness of the knees, elbows, and neck but no joint swelling. The sputum was blood-stained.

Her chest radiographs showed a cavity containing a fluid level in the posterior segment of the right upper lobe with a zone of partial consolidation and collapse. There were also ill-defined opacities in the left upper lobe. The sputum contained numerous pus cells, but culture grew only normal commensal organisms. Ten sputa were negative for $\mathrm{Myco-}$ bacterium tuberculosis. Blood cultures were negative. Haemoglobin 10.8 g./ $1 \mathrm{CO} \mathrm{ml}$., W.B.C. $12,700 /$ c.mm., $79 \%$ polymorphs and $1 \%$ eosinophils; Rose-Waaler positive $1: 1,028$; latex fixation test negative. Urine analysis was normal. Neither malignant cells nor fungi were found on repeated examination of the sputum.

Treatment with penicillin, 2 mega units six-hourly, was commenced but the fever showed no response. Further consolidation of the right upper lobe occurred with an increase in the size of the cavity, and consolidation spread rapidly through the left upper lobe (Fig. 3). The patient's condition deteriorated. Treatment was changed to streptomycin, 1 g. b.d., and chloramphenicol, $500 \mathrm{mg}$. q.d.s. There was no improvement and the lesions in the chest continued to increase in size. She remained pyrexial, coughing up purulent heavily blood-stained sputum. Empirical treatment with prednisone, $40 \mathrm{mg}$. daily, was commenced, and immediately there was some improvement with a fall in temperature and the production of less sputum. Within two days there was appreciable clearing of the consolidation of the right upper lobe. However, this improvement was only transitory and she deteriorated again rapidly. The consolidation extended to involve the whole of the right lung and practically the whole of the left. Albumin, red blood cells, and granular casts appeared in the urine and the blood urea rose to $360 \mathrm{mg} . / 100 \mathrm{ml}$. The sputum became very blood-stained again and she passed some blood per vaginam and per rectum. Bleeding and clotting times were normal. She appeared to be in extremis.

A red papular eruption occurred at this time on the extensor surface of the elbows and buttocks. Biopsy showed necrotizing lesions within several small arteries of the dermis associated with an acute and chronic inflammatory cell reaction (Fig. 4).
It was presumed that she had a widespread necrotizing arteritis affecting the lungs, kidney, skin, and gastro-intestinal and genital tracts, and in view of this the dose of prednisone was increased to $100 \mathrm{mg}$. daily. The temperature fell to normal and the areas of consolidation began to lessen. After several days she was much better but began to experience severe epigastric pain and passed a melaena stool. Two pints of blood were transfused. Prednisone was reduced to $70 \mathrm{mg}$. daily and the haemoptysis and chest radiograph appearances continued to improve.

Improvement in her general condition was maintained over the next few weeks, but she continued to show proteinuria. She was discharged from hospital three months after admission taking $30 \mathrm{mg}$. prednisone daily. The chest film showed almost complete resolution of the lesions in both upper lobes (Fig. 5). She had moderate proteinuria with a few red blood cells. Blood urea $50 \mathrm{mg}$. $/ 100 \mathrm{ml}$.; creatinine clearance $13.6 \mathrm{ml} . / \mathrm{min}$. ; average ward blood pressure $140 / 90$ $\mathrm{mm} . \mathrm{Hg}$; Rose-Waaler negative.

She has remained well and active for four years. At present she takes $20 \mathrm{mg}$. prednisone each day, and her haemoglobin is $13.7 \mathrm{~g} . / 100 \mathrm{ml}$. No radiographic abnormality of the nasal sinuses has ever been demonstrated. Several small infiltrative lesions have appeared from time to time in the lungs but have cleared after the dose of steroids has been temporarily increased. There is no evidence of airway obstruction, but the lung volumes are moderately reduced; the total lung capacity is 3.991 . (normal 4.781 .) and vital capacity 2.511 . (normal $3 \cdot 131$.). The carbon monoxide diffusing capacity is reduced from a predicted 21.6 to $8.8 \mathrm{ml}$./ $\mathrm{min} . / \mathrm{mm}$. Hg. Rose-Waaler positive 1:64; antinuclear factor negative; resting blood pressure 150/ $100 \mathrm{~mm}$. $\mathrm{Hg}$; blood urea $56 \mathrm{mg} . / 100 \mathrm{ml}$.; creatinine clearance $48.5 \mathrm{ml} . / \mathrm{min}$.

Renal biopsy (Fig. 6) 'The sections show cortical tissue with numerous glomeruli. The majority of the glomeruli show varying degrees of hyalinization and fibrosis. Rounded masses of fibrosis are present in some. Only an occasional glomerulus is spared. There is a marked increase in interstitial tissue with many atrophic tubules, a few containing protein. Small scattered foci of chronic inflammatory cells are present, mainly composed of lymphocytes together with a few plasma cells. Periglomerular and peritubular fibrosis are prominent. Arteriosclerosis is moderate. No necrotizing arteritis or capillaritis is present. The histology is that of a chronic glomerulonephritis of indeterminate aetiology.'

\section{DISCUSSION}

The criteria of Godman and Churg (1954) for Wegener's granulomatosis are (a) necrotizing granulomatous lesions of the upper and/or lower respiratory tract, (b) generalized necrotizing vasculitis, and (c) focal glomerulitis. The diagnosis is basically a histological one and a biopsy must be obtained. Upper respiratory tract granulomata, 
when present, form the usual source of material for histology, but kidney (Muehrcke and Pirani, 1962), skin (Walton, 1958), lung, synovial membrane (Kinney, Olsen, Hepper, and Harrison, 1961), bronchus (Beidleman, 1963), and muscle (Rose and Spencer, 1957) have also been used. Both cases described here presented as an acute illness with cavitating lesions in the lungs associated with massive haemoptysis, rapid deterioration in health, fever, and leucocytosis. In case 1 the diagnosis of Wegener's granulomatosis was not revealed until necropsy. However, the urine contained red cells and protein, and in retrospect the glomerulitis demonstrated after death might have been detected in life by renal biopsy. Also the peripheral nodules seen on the chest radiographs might have suggested scattered granulomata. In case 2 a skin biopsy showed a necrotizing arteritis, and the diagnosis was further substantiated by the appearance of protein, red cells, and granular casts in the urine. The renal biopsy performed after two years' continuous steroid therapy showed no evidence of vasculitis, only extensive glomerular hyalinization and destruction with interstitial fibrosis, identical to the changes of chronic glomerulonephritis. This appearance lacks specificity but does not refute the diagnosis. It is perhaps to be expected, for the disseminated vasculitis and pulmonary granulomata have disappeared clinically and radiologically. Similar non-specific changes are well described in the kidneys in polyarteritis nodosa after the acute stage has subsided (Harvey, Walker, and Yardley, 1963).

Although the clinical features are protean, Wegener's granulomatosis is a distinct clinicopathological entity. It is usually regarded as a chronic illness with an average duration of five to six months before death, but in some patients a limited form of the disease may occur with a better prognosis (Carrington and Liebow, 1966). Upper respiratory symptoms, such as rhinorrhoea and swelling over the nasal sinuses, are typically present, but in about one-third symptoms are restricted to the lower respiratory tract. These two cases are recorded because they show an unusually rapid course proceeding to an almost terminal stage in only 10 to 14 days and they emphasize that the diagnosis must be considered with some urgency if treatment is to be of any value. Budzilovich and Wilens (1960) recorded a fulminating case of three weeks' duration, but respiratory symptoms and signs were not prominent. Lindsay, Aggeler, and Lucia (1944) record one case of only four weeks' duration. Walton (1958) and Fahey, Leonard, Churg, and Godman (1954) each mention one case of similar rapidity and stress that the diagnosis should be considered whenever pulmonary cavitation and haemoptysis occur which are not due to tuberculosis or neoplasm and do not respond to antibiotics. These two cases show that the suggestion is still applicable even when such cavitation occurs very rapidly.

Fred, Lynch, Greenberg, and Gonzalez-Angulo (1964) collected from the literature 31 cases of Wegener's granulomatosis treated with cortico- or steroids before the terminal phase of the illness, $\vec{\omega}$ and, of these, 22 gained temporary improvement. Carrington and Liebow (1966) described $10 \stackrel{\circ}{\leftrightharpoons}$ patients treated with steroids, four of whom $\vec{c}$ appeared to improve. Both Kinney et al. (1961) and Beidleman (1963) described patients in whom moderated doses of prednisone (20 to $40 \mathrm{mg}$.) produced a dramatic improvement within 24 hours. Berman, Rydell, and Eichenholz (1963) reported $\vec{\oplus}$ considerable improvement in two of three patients of treated with moderate doses of steroids. One patient with oliguria, a blood urea of $235 \mathrm{mg}$. / 100 ml., and pericarditis improved dramatically after $350 \mathrm{mg}$. hydrocortisone given in 24 hours followed by a gradual reduction in dosage. \% Empirical treatment with $15 \mathrm{mg}$. prednisone in $\stackrel{\mathbb{Q}}{2}$ our first case was ineffective. In our second case, $\overrightarrow{\vec{O}}$ $40 \mathrm{mg}$. prednisone produced only a temporary 3 improvement ; $100 \mathrm{mg}$. prednisone was required $\rightleftharpoons$ to obtain a satisfactory remission, and the response was dramatic. Despite adequate control of the acute respiratory lesions and suppression of the generalized necrotizing arteritis the renal $\stackrel{\infty}{x}$ dysfunction resulting in hypertension and albu- $\frac{3}{3}$ minuria has not remitted, and renal biopsy indicates that the kidneys are irreversibly damaged by chronic glomerulonephritis. Pulmo-

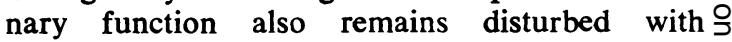
evidence of impaired gas transfer. However, the $D$ rapid control of the acute symptoms and the relative well-being of the patient over the last $N$ four years have made treatment worth while.

The presence of the rheumatoid factor in high concentrations in case 2 raises the possibility of a relationship between Wegener's granulomatosis? and rheumatoid arthritis. After reviewing theo relationship of arteritis in the different collagen- $\mathbb{D}$ oses Alarcón-Segovia and Brown (1964) con-? cluded that the angiitis of rheumatoid arthritis $\frac{T}{0}$ differed from that of Wegener's granulomatosis $\frac{\vec{D}}{\mathbb{D}}$ and polyarteritis nodosa. However, Berman et al. $\stackrel{?}{\overparen{\Phi}}$ (1963) recorded chronic inflammatory changes $\varrho$ similar to those of rheumatoid arthritis in the 
synovial biopsy of a patient with Wegener's granulomatosis but did not report the rheumatoid factor. Although tests for rheumatoid factor may be positive in interstitial pulmonary fibrosis (Turner-Warwick and Doniach, 1965) the presence of this factor has been described in only one other patient with Wegener's granulomatosis (Carrington and Liebow, 1966). However, in this case there was prior clinical and laboratory evidence of lupus erythematosus and rheumatoid arthritis. It would be worth seeking in all cases, for it may assist the diagnosis, if present, and may also contribute to the aetiology of this obscure condition.

We are indebted to Dr. James Livingstone and Dr. J. C. H. MacKenzie for their interest and to the Departments of Pathology at King's College Hospital and Groby Road Hospital. The renal biopsy was performed by Dr. H. A. Lee.

\section{REFERENCES}

Alarcón-Segovia, D., and Brown, A. L. (1964). Classification and etiologic aspects of necrotizing angiitides. Mayo Clin. Proc., 39, 205.
Beidleman, B. (1963). Wegener's granulomatosis-prolonged therapy with large doses of steroids. J. Amer. med. Ass., 186, 827.

Berman, D. A., Rydell, R. E., and Eichenholz, A. (1963). Wegener's granulomatosis-a clinico-pathologic study of four cases. Ann. intern. Med., 59, 521.

Budzilovich, G. N., and Wilens, S. L. (1960). Fulminating Wegener's granulomatosis. Arch. Path., 70, 653.

Carrington, C. B., and Liebow, A. A. (1966). Limited forms of angiitis and granulomatosis of Wegener's type. Amer. J. Med., $41,497$.

Fahey, J. L., Leonard, E., Churg, J., and Godman, G. (1954). Wegener's granulomatosis. Ibid., 17, 168.

Fred, H. L., Lynch, E. C., Greenberg, S. D., and Gonzalez-Angulo, A. (1964). A patient with Wegener's granulomatosis exhibiting unusual clinical and morphologic features. Ibid., 37, 311.

Godman, G. C., and Churg, J. (1954). Wegener's granulomatosispathology and review of the literature. Arch. Path., 58, 533.

Harvey, A. M., Walker, W. G., and Yardley, J. H. (1963). In Diseases of the Kidney p. 603, ed. M. B. Strauss and L. G. Welt. Churchill, London.

Kinney, V. R., Olsen, A. M., Hepper, N. G. G., and Harrison, E. G. (1961). Wegener's granulomatosis-report of two cases and brief review. Arch. intern. Med., 108, 269.

Lindsay, S., Aggeler, P. M., and Lucia, S. P. (1944). Chronic granuloma associated with periarteritis nodosa. Amer. J. Path. 20, 1057.

Muehrcke, R., and Pirani, C. L. (1962). In Renal Disease, ed. D. A. K. Black, p. 418. Blackwell, Oxford.

Rose, G. A., and Spencer, H. (1957). Polyarteritis nodosa. Quart. J. Med., 26, 43.

Turner-Warwick, M., and Doniach, D. (1965). Auto-antibody studies in interstitial pulmonary fibrosis. Brit. med. J., 1, 886.

Walton, E. W. (1958). Giant-cell granuloma of the respiratory tract (Wegener's granulomatosis). Ibid., 2, 265.

Wegener, F. (1936). Ubber generalisierte, septische Gefässerkrankungen. Verh. dtsch. path. Ges., 29, 202. 